\title{
Limited-angle tomography applied to biological objects
}

\author{
Wojciech Krauze*, Małgorzata Kujawińska \\ Institute of Micromechanics and Photonics, Warsaw University of Technology, sw. A. Boboli 8, 02-525 Warszawa,
}

Received October 22; accepted November 12, 2013; published December 31, 2013

\begin{abstract}
Optical limited-angle tomography is strongly required in biological applications, as often there is access to projections gathered from a small angle range. Choosing the reconstruction algorithm among many available is always a trade-off between the speed of calculation and the quality of reconstruction. In this paper we present the studies how a priori information about an object's geometry influences the efficiency of the Data Replenishment Algorithm. The analysis is based on tomographic reconstruction of a real biological cell of human malignant lymphoma.
\end{abstract}

Optical tomography is a dynamically evolving field of science which allows three-dimensional imaging of external geometry and internal structures of a semitransparent object. Compared to optical holography, it gives significantly more extensive spatial frequency coverage [1]. It happens so because in holography we record a complex field propagated across the object in one direction only, whereas in tomography we record tens (or even hundreds) of such fields over different angles. In most cases, by rotating an object, we can get its projections from $180^{\circ}$. In that case, in the Fourier domain the spatial frequencies are isotropically filled and cover the whole spectrum (see Projection Slice Theorem [2]). This results in proper reconstruction as shown in Fig.1. However, there are many situations when the angle range is limited. It happens when, for example, we measure biological cells in a Petri dish. In such a case, an object is reconstructed from those projections that can be safely reached. Unlike in the $180^{\circ}$ case, in the Fourier domain there are now empty areas where no information about an object's spatial frequencies is available.

There are two ways to overcome the problem of a highly limited angle range of projections: (1) modifying the tomographic setup or (2) applying special numerical algorithms. The former relates to changes in the method of recording projections. This allows providing additional spatial frequencies [3-4]. The latter solution requires implementing a specific reconstruction algorithm. Standard tomographic algorithms, like Filtered Back Projection, have serious difficulties when reconstructing an object from a limited number of projections. There happens no replenishment of empty areas of a spectrum. As a result, we get characteristically distorted images (see Fig. 2 obtained for the angle of projections limited to $90^{\circ}$ around direction "1"). The last 30 years resulted in many

\footnotetext{
*E-mail: w.krauze@mchtr.pw.edu.pl
}

different ways of overcoming this problem [5-9]. All of these new methods are computationally more demanding and require more time to run than their full-angle equivalents. It happens so because most of them work in the iterative way. One of the fastest and easiest to implement is the Data Replenishment (DR) Algorithm [5]. However, it may lead to erroneous results.

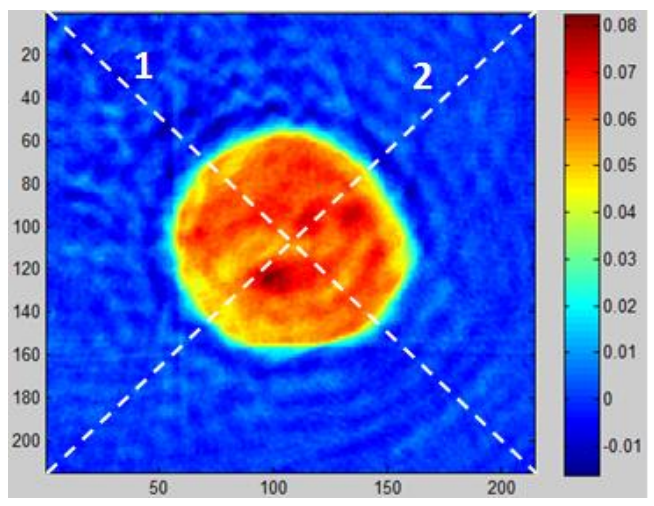

Fig. 1. Reconstruction of an exemplary layer of a HML cell calculated with standard Back Projection Algorithm from $180^{\circ}$. This reconstruction will be treated as reference in further analysis. Two cross-sections $(1,2)$ which will be used later, are marked. $Q=1$.

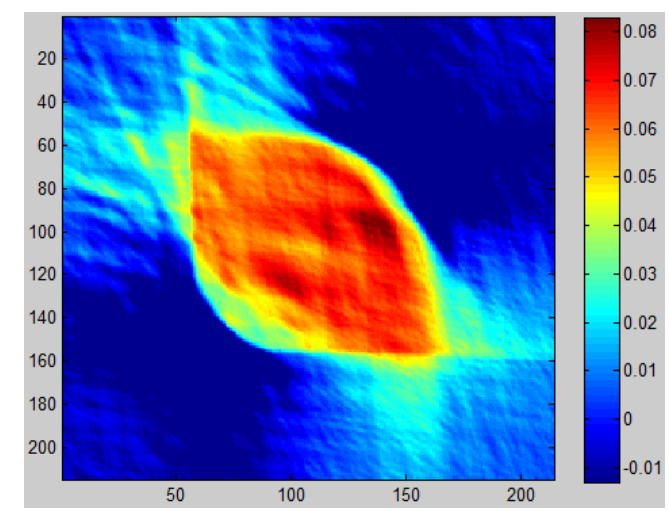

Fig. 2. A layer of the cell reconstructed with the standard version of Back Projection algorithm, $Q=0.7091$.

In the case of some samples, we can partially reduce this disadvantage by applying a priori knowledge about an object e.g. round external geometry, smoothness of the inner refractive index distribution or known minimal 
refractive index value. The example of such an object, which is further taken for our consideration and simulation, is a round human malignant lymphoma (HML) cell (Fig. 1).

In this paper, we prove that in the case of such objects, applying the mask with external geometry is sufficient to accelerate tomographic algorithms and improve the quality of a reconstructed image.

Surprisingly, one of the most difficult tasks in testing a tomographic algorithm is the assessment of its efficiency. In the case of numerical phantom (e.g. Shepp-Logan), the reconstructed function is compared with the original model. When the object is a real sample, like a biological cell, then the reconstruction from a limited angle range $Y$ is compared with the full angle case $\mathrm{X}$. For this purpose we have implemented an Universal Image Quality Index [10]. It works as a combination of correlation loss, luminance distortion and contrast distortion. The mathematical formula describing this Index is:

$$
Q=\frac{4 \sigma_{x y} \bar{x} \bar{y}}{\left(\sigma_{x}^{2}+\sigma_{y}^{2}\right)\left(\bar{x}^{2}+\bar{y}^{2}\right)}
$$

where

$$
\begin{gathered}
\sigma_{x y}=\frac{1}{N-1} \sum_{i=1}^{N}\left(x_{i}-\bar{x}\right)\left(y_{i}-\bar{y}\right) \\
\sigma_{x}^{2}=\frac{1}{N-1} \sum_{i=1}^{N}\left(x_{i}-\bar{x}\right)^{2}
\end{gathered}
$$

where $\mathrm{X}$ and $\mathrm{Y}$ are the two compared matrices, and $\bar{x}, \bar{y}$ are mean the values of $\mathrm{X}$ and $\mathrm{Y}$, respectively. The resemblance of the two matrices is given in the range of $<-1,1>$, where 1 stands for two equal matrices. In this iterative algorithm, the quality of reconstruction increases until reaching a maximum value. After that point the image gets only more distorted. So the final result is the iteration with the highest $Q$.

In our tests, the DR Algorithm is modified as shown in Fig. 3. To start the program, some initial data is required. This data is the set of projections from an available angle range arranged in the form of a sinogram. The first step in the algorithm is to calculate the inverse Radon transform from the sinogram. This reconstruction results in a distorted image, in which the level of distortion depends on the range of the angle. At this point we need to analyze the a priori knowledge about the object. The first question is whether the phase of our object can be described with a slowly varying function. If so, then this $a$ priori information is realized in the algorithm by applying median filtering in the reconstruction domain. The second question is whether our object has a known and symmetrical external shape. In the case of the HML cell, we can implement a round mask at this point. After these operations we calculate a Radon transform for all angles (180 ${ }^{\circ}$ angle range). As a result, we get a new sinogram, which is extended, when compared to the first sinogram, by projections from an unknown region. Even in the known region, the sinogram looks now different. Then we replenish the data in the known region with the first data set. The rest of the sinogram stays unchanged. Such a new, hybrid sinogram is now subjected to filtering.

All these steps create one iteration. Usually, after the first iteration the distortion of the reconstruction is significantly reduced. Every next iteration improves the quality of the reconstruction to a smaller extent.

In order to assess the impact of the mask with the external geometry of the object on the quality of reconstruction, we performed a test of the DR Algorithm. In our case the projections of an HML cell were gathered with Digital Holographic Microscopy in the tomographic setup [11]. In principle, we had data from $180^{\circ}$. To test the procedure with the angle of projections limited to $90^{\circ}$, we had cut the full sinogram of our object by half. Later we calculated the reconstruction of the HML with and without a geometry mask, and compared the results.

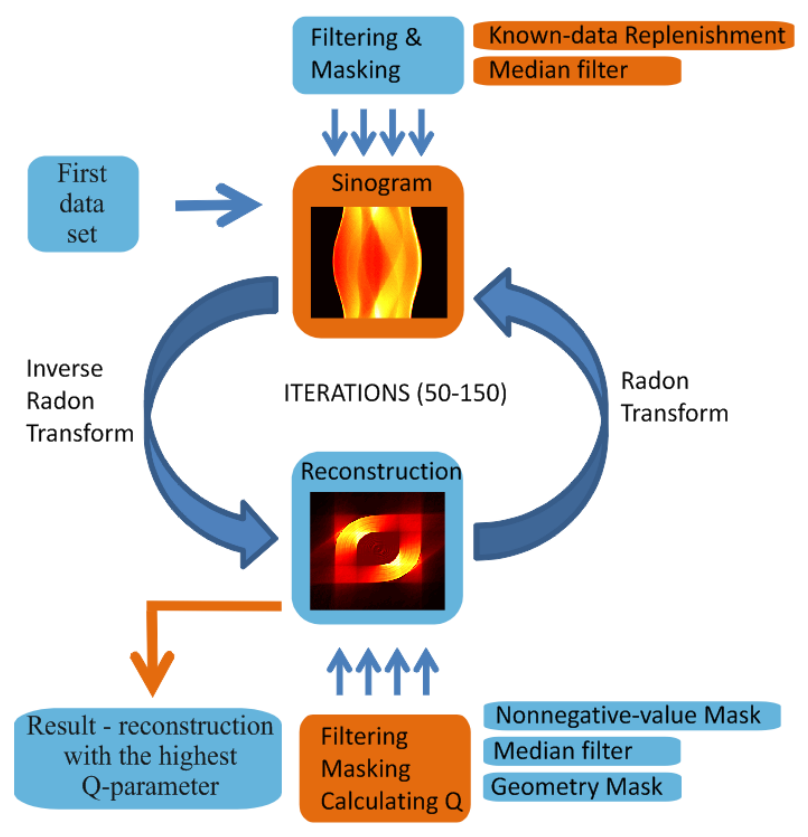

Fig. 3. The basis of the DR Algorithm.

To find the best reconstruction out of the series of images, every reconstruction in the algorithm is compared to the full reconstruction (from an uncut sinogram) calculated with a Filtered Back Projection algorithm.

In Fig. 4 we present the reconstruction calculated by means of a DR Algorithm with the geometry mask applied. In Fig. 5 the reconstruction without a mask is shown. In order to find the best reconstruction in both cases, we calculated 100 iterations, and chose the one with the highest $Q$ parameter. 


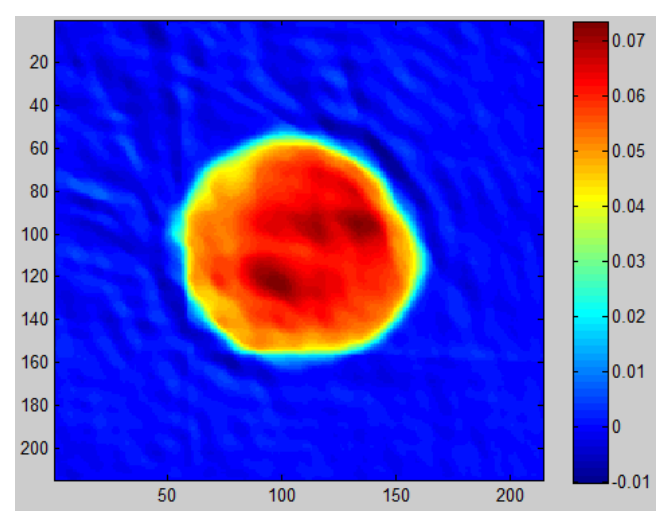

Fig. 4. Reconstructions calculated with a DR Algorithm with a geometry mask applied. $Q=0.9891$. Iteration no. 13 .

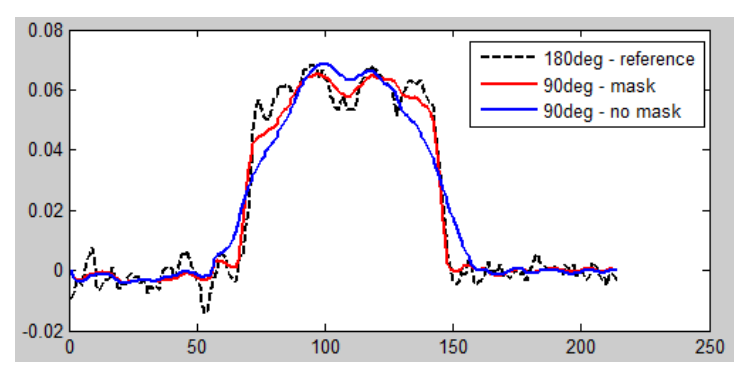

Fig. 6. Comparison of the diagonal cross-section of the reconstructed image from Figs. 4-5 and the ideal reconstruction from Fig. 1 calculated alond the cross-section marked as "1" in Fig. 1.

From the results we conclude that using a DR Algorithm improves the quality of reconstruction when the angle range of projections is limited, regardless of whether we use a geometry mask or not.

When comparing two reconstructions from Fig. 4 and Fig. 5, the impact of an additional geometry mask applied to the cell is clearly visible. The improvement is expressed not only by the higher quality of the final reconstruction (0.9891) than without a mask (0.9761), but also by fewer iterations necessary (13 vs. 22) to achieve the best reconstruction.

With a geometry mask applied, even when half the data is missing, the result computed with a DR Algorithm is very close to the full angle $\left(180^{\circ}\right)$ case. The shape of the cell is now almost ideal and, the internal structures are better reconstructed (Fig. 6 and Fig. 7). We can observe 40\% improvement in parameter $Q(0.9891$ from Fig. 4 compared to 0.7091 from Fig. 2). It must be mentioned that it is not quantitatively meaningful because image quality is a subjective type of criteria that is hard to quantify. However, in our test, such improvement in $Q$ is consistent with our subjective observation of reconstructions. We proved that for objects with a symmetric shape, especially a round one, it is worth implementing a mask with that shape. It decreases the required number of iterations - thus decreasing the computational time of an algorithm.

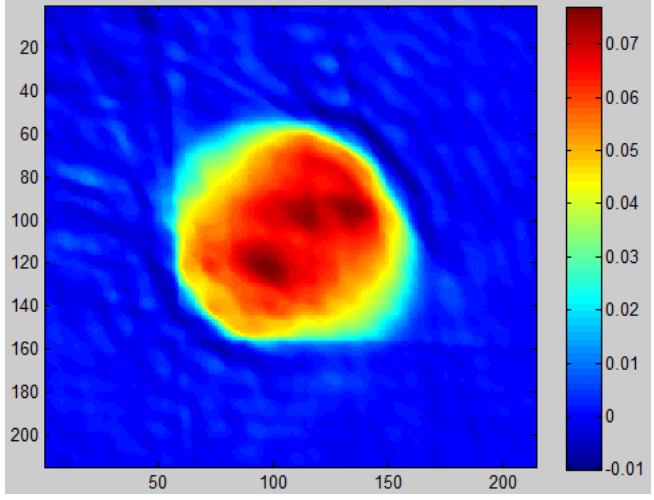

Fig. 5. Reconstructions calculated with a DR Algorithm without a mask. $Q=0.9761$. Iteration no. 22 .

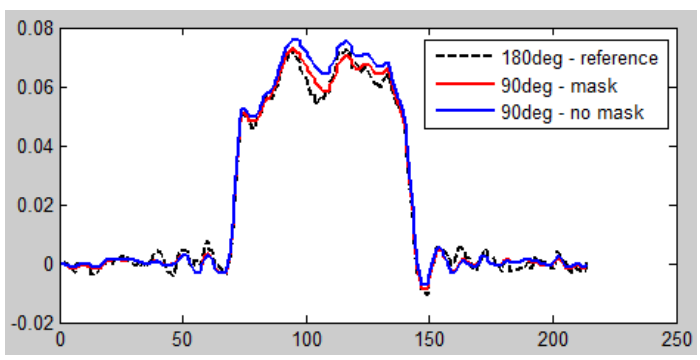

Fig. 7. Comparison of the diagonal cross-section of the reconstructed image from Figs. 4-5 and the ideal reconstruction from Fig. 1 calculated along the cross-section marked as "2" in Fig. 1.

This work was partly founded by the project TEAM/2011-7/7 of the Foundation for Polish Science, co-financed from the European Funds of Regional Development. It was also co-financed by the grant of the Dean of Mechatronics Faculty, Warsaw University of Technology. The authors acknowledge Mr. A. Kuś, Mr. M. Dudek and Dr. B. Kemper for providing projection data of a human malignant lymphoma cell which were collected at the University of Munster.

\section{References}

[1] S. Kou, C. Sheppard, Opt. Lett. 33, 2362 (2008)

[2] J. Hsieh, Computed Tomography Principles, Design, Artifacts, and Recent Advances (Wiley 2009).

[3] S. Isikman et al., P. Natl. Acad. Sci. USA 108, 7296 (2011).

[4] S. Vertu, E. Maeda, J. Flügge, J. Delaunay, O. Haeberlé, Proc. SPIE 7904, 790410 (2011).

[5] A. Likhachov, Optoelectron. Instrum. Data Process. 45, 55 (2009).

[6] R. Gordon, IEEE T. Nucl. Sci. 21, 78 (1974).

[7] A. Andersen, A. Kak, Ultrasonic Imaging 6, 81 (1984)

[8] D. Verhoeven, Appl. Optics 32, 3736 (1993).

[9] J. Fessler, B. Sutton, IEEE T. Signal Proces. 51, 560 (2003).

[10] Z. Wang, A. Bovik, IEEE Signal Proc. Let. 9, 81 (2002).

[11] M. Kujawińska et al., Fringe 2013, 671 (2013). 